\title{
Differentiation syndrome in patients with acute promyelocytic leukemia treated with all-trans retinoic acid and anthracycline chemotherapy: characteristics, outcome, and prognostic factors
}

\author{
Pau Montesinos, ${ }^{1,2}$ Juan M. Bergua, ${ }^{3}$ Edo Vellenga, ${ }^{4}$ Chelo Rayón, ${ }^{5}$ Ricardo Parody, ${ }^{6}$ Javier de la Serna, ${ }^{7}$ Angel León, ${ }^{8}$ \\ Jordi Esteve, ${ }^{9}$ Gustavo Milone, ${ }^{10}$ Guillermo Debén, ${ }^{11}$ Concha Rivas, ${ }^{12}$ Marcos González, ${ }^{13}$ Mar Tormo, ${ }^{14}$ \\ Joaquín Díaz-Mediavilla, ${ }^{15}$ Jose D. González, ${ }^{16}$ Silvia Negri, ${ }^{17}$ Elena Amutio, ${ }^{18}$ Salut Brunet, ${ }^{19}$ Bob Lowenberg, ${ }^{20}$ and \\ Miguel A. Sanz ${ }^{1}$
}

${ }^{1}$ Hospital Universitario La Fe, Valencia, Spain; ${ }^{2}$ Department of Medicine of the Universitat Autònoma de Barcelona, Barcelona, Spain; ${ }^{3}$ Hospital San Pedro de Alcántara, Cáceres, Spain; ${ }^{4}$ University Hospital, Groningen, The Netherlands; ${ }^{5}$ Hospital Central de Asturias, Oviedo, Spain; ${ }^{6}$ Hospital Universitario Virgen del Rocío, Sevilla, Spain; ${ }^{7}$ Hospital 12 de Octubre, Madrid, Spain; ${ }^{8} \mathrm{Hospital}$ General, Jerez de la Frontera, Spain; ${ }^{9} \mathrm{Hospital}$ Clinic, Barcelona, Spain; ${ }^{10}$ Fundaleu, Buenos Aires, Argentina; ${ }^{11} \mathrm{Hospital}$ Juan Canalejo, La Coruña, Spain; ${ }^{12} \mathrm{Hospital}$ General, Alicante, Spain; ${ }^{13} \mathrm{Hospital} \mathrm{Universitario,} \mathrm{Salamanca,} \mathrm{Spain;}{ }^{14} \mathrm{Hospital}$ Clínico Universitario, Valencia, Spain; ${ }^{15} \mathrm{Hospital}$ Clínico San Carlos, Madrid, Spain; ${ }^{16} \mathrm{Hospital}$ Insular, Las Palmas, Spain; ${ }^{17}$ Hospital Carlos Haya, Málaga, Spain; ${ }^{18} \mathrm{Hospital}$ de Cruces, Baracaldo, Spain; ${ }^{19} \mathrm{Hospital}$ Sant Pau, Barcelona, Spain; and ${ }^{20}$ Erasmus University Medical Center, Rotterdam, The Netherlands

\begin{abstract}
Differentiation syndrome (DS) can be a life-threatening complication in patients with acute promyelocytic leukemia (APL) undergoing induction therapy with alltrans retinoic acid (ATRA). Detailed knowledge about DS has remained limited. We present an analysis of the incidence, characteristics, prognostic factors, and outcome of 739 APL patients treated with ATRA plus idarubicin in 2 consecutive trials (Programa Español de Tratamientos en Hematología [PETHEMA] LPA96 and
\end{abstract}

LPA99). Overall, 183 patients $(24.8 \%)$ experienced DS, 93 with a severe form $(12.6 \%)$ and 90 with a moderate form (12.2\%). Severe but not moderate DS was associated with an increase in mortality. A bimodal incidence of DS was observed, with peaks occurring in the first and third weeks after the start of ATRA therapy. A multivariate analysis indicated that a WBC count greater than $5 \times 10^{9} / \mathrm{L}$ and an abnormal serum creatinine level correlated with an increased risk of developing severe
DS. Patients receiving systematic prednisone prophylaxis (LPA99 trial) in contrast to those receiving selective prophylaxis with dexamethasone (LPA96 trial) had a lower incidence of severe DS. Patients developing severe DS showed a reduced 7-year relapse-free survival in the LPA96 trial $(60 \%$ vs $85 \%, P=.003)$, but this difference was not apparent in the LPA99 trial ( $86 \%$ vs $88 \%$ ). (Blood. 2009;113:775-783)

\section{Introduction}

Differentiation syndrome (DS), formerly known as retinoic acid syndrome, can be a life-threatening complication in patients with acute promyelocytic leukemia (APL) undergoing induction therapy with all-trans retinoic acid (ATRA) or arsenic trioxide. The full-blown syndrome is characterized by unexplained fever, weight gain, peripheral edema, dyspnea with interstitial pulmonary infiltrates, pleuropericardial effusion, hypotension, and acute renal failure. ${ }^{1}$ The reported incidence of this syndrome of uncertain pathogenesis ranges from $2 \%$ to $27 \% .^{1-10}$ This wide range is probably because of the different criteria used for the diagnosis of DS, and to the effects on the incidence and severity of the syndrome due to differences in induction therapy and supportive measures. In this regard, the early addition of chemotherapy to ATRA and the administration of high-dose dexamethasone at the onset of the first symptoms appear to have reduced the DS-related mortality to $1 \%$ or less in the most recent trials. ${ }^{9-11}$

Although the mortality associated with DS is now relatively low, the development of this complication has additional implications, such as an increase in morbidity leading to an increased use of hospital resources, and it has been suggested that this is associated with an increased risk of relapse independent of the white blood cell (WBC) count. ${ }^{11}$ However, this interesting finding reported by the European APL Group has not yet been confirmed in other studies.

Apart from a peak WBC count frequently observed at the onset of DS symptoms, ${ }^{2}$ very little is known about other factors predictive of DS when patients are treated with ATRA combined with chemotherapy. In this context, only one published study included a significant number of patients and examined possible prognostic factors, including pretreatment WBC count, associated with the development of DS. ${ }^{10}$ This study found that none of the examined variables had prognostic value. Furthermore, the Programa Español de Tratamientos en Hematología (PETHEMA) group has recently analyzed the causes and prognostic factors of induction failure, including death due to DS, but the incidence, time of occurrence, and prognostic factors of the development of this syndrome were not analyzed. ${ }^{12}$

This study was designed to analyze the incidence, clinical manifestations, and prognostic factors associated with DS, as well as the short- and long-term outcomes for patients developing this syndrome in a large series of patients with newly diagnosed APL treated with ATRA and idarubicin (AIDA regimen) who were
Submitted July 14, 2008; accepted September 18, 2008. Prepublished online as Blood First Edition paper, October 22, 2008; DOI 10.1182/blood-2008-07-168617.

The online version of this article contains a data supplement.
The publication costs of this article were defrayed in part by page charge payment. Therefore, and solely to indicate this fact, this article is hereby marked "advertisement" in accordance with 18 USC section 1734.

() 2009 by The American Society of Hematology 
enrolled in 2 consecutive studies by the PETHEMA Group (LPA96 and LPA99). We also analyzed the impact of the different regimens of corticosteroid prophylaxis used in these trials on the development and severity of DS.

\section{Methods}

\section{Eligibility}

For enrolment in the consecutive PETHEMA LPA96 and LPA99 trials, patients were required to have a diagnosis of de novo APL with demonstration of the $\mathrm{t}(15 ; 17)$ or promyelocytic leukemia-retinoic acid receptor- $\alpha$ $(P M L-R A R A)$ rearrangements, normal hepatic and renal function, no cardiac contraindications to anthracycline chemotherapy, and an Eastern Cooperative Oncology Group (ECOG) performance status of less than 4. Informed consent was obtained from all patients in accordance with the Declaration of Helsinki. The protocol was approved by the Research Ethics Board of each participating hospital.

\section{Induction therapy}

Treatment with ATRA was started as soon as a diagnosis of APL by morphologic criteria was made. ${ }^{13,14}$ Induction therapy consisted of oral ATRA (45 mg/m $\mathrm{m}^{2}$ per day) divided into 2 daily doses, which was maintained until complete hematologic remission or for a maximum of 90 days, and idarubicin $\left(12 \mathrm{mg} / \mathrm{m}^{2}\right.$ per day) given as an intravenous bolus on days 2, 4, 6, and 8 (AIDA regimen). For patients 20 years of age or younger, the ATRA was adjusted to $25 \mathrm{mg} / \mathrm{m}^{2}$ per day. Since November 1999 (LPA99 trial), the dose of idarubicin on day 8 has been omitted for patients more than 70 years of age.

\section{Postremission therapy}

Details of the consolidation and maintenance therapy have been described elsewhere. ${ }^{15}$ Briefly, the first consolidation course consisted of idarubicin $\left(5 \mathrm{mg} / \mathrm{m}^{2}\right.$ per day for 4 days), the second of mitoxantrone $\left(10 \mathrm{mg} / \mathrm{m}^{2}\right.$ per day for 5 days), and the third of idarubicin $\left(12 \mathrm{mg} / \mathrm{m}^{2}\right.$ per day for 1 day). From November 1999 (LPA99 study), intermediate- and high-risk patients, as previously defined, ${ }^{16}$ received ATRA $\left(45 \mathrm{mg} / \mathrm{m}^{2}\right.$ per day for 15 days) combined with the reinforced single-agent chemotherapy courses. Patients who tested negative for $P M L-R A R A$ at the end of consolidation were started on maintenance therapy with oral mercaptopurine $\left(50 \mathrm{mg} / \mathrm{m}^{2}\right.$ per day), intramuscular methotrexate $\left(15 \mathrm{mg} / \mathrm{m}^{2}\right.$ per week $)$, and oral ATRA ( $45 \mathrm{mg} / \mathrm{m}^{2}$ per day for 15 days every 3 months) over 2 years.

\section{Management of DS and other supportive measures}

Treatment with intravenous dexamethasone at a dose of $10 \mathrm{mg}$ twice daily was started promptly at the very earliest symptom or sign of DS. ATRA was discontinued in cases where there was progression of the clinical symptoms of DS. In the LPA96 trial, patients with a WBC count greater than $5 \times 10^{9} / \mathrm{L}$ (before or during treatment with ATRA) received prophylaxis with dexamethasone $(10 \mathrm{mg} / 12$ hours intravenously for 7 days), while in the LPA99 study all patients, regardless of WBC count, received DS prophylaxis with prednisone $(0.5 \mathrm{mg} / \mathrm{kg}$ per day orally) from days 1 through 15. Diuretics were usually administered to treat acute renal failure and weight gain, and dialysis or venous continuous ultrafiltration was performed at the physicians' discretion. Mechanical ventilation was indicated in some patients with severe acute respiratory failure. Details about the management of coagulopathy, transfusion policy, and other supportive measures have been described elsewhere. ${ }^{12}$

\section{Study definitions and endpoints}

The remission induction response was assessed according to the criteria recently revised by Cheson et $\mathrm{al}^{17} \mathrm{~A}$ morphologic complete remission (CR) designation requires a patient to have less than $5 \%$ blasts and atypical promyelocytes in an aspirate sample, an absolute neutrophil count of more than $10^{9} / \mathrm{L}$ and a platelet count of more than $100 \times 10^{9} / \mathrm{L}$. The treatment failure group included those patients for whom treatment had failed to achieve a CR. The primary end point of the study was the incidence of DS. Diagnosis of this syndrome was made according to the presence of the following signs and symptoms described by Frankel et $\mathrm{l}^{1}$ : dyspnea, unexplained fever, weight gain greater than $5 \mathrm{~kg}$, unexplained hypotension, acute renal failure, and, particularly, a chest radiograph demonstrating pulmonary infiltrates or pleuropericardial effusion. Patients with 4 or more of the above signs or symptoms were classified as having severe DS, while those with 2 or 3 signs or symptoms were considered to have moderate DS. No single sign or symptom was considered sufficient to make a diagnosis of the syndrome. Patients with alternative explanations for the clinical complex, such as pulmonary hemorrhage, septic shock, pneumonia, or cardiac failure, were considered to not have DS. Death was attributed to DS when death occurred in patients with severe DS and was not explained by infection, hemorrhage, or other causes. DS was defined as "early" when it occurred within 7 days of the start of ATRA, or "late" when it occurred more than 7 days after the start of ATRA.

\section{Data collection and prognostic factors}

Data were collected and registered prospectively. Thirty-six patient and disease characteristics documented at the initial evaluation were examined for prognostic factor analysis to establish their relationship to the development of DS. Basic demographic data and clinical characteristics at presentation included age, sex, ECOG score, fever, total body surface, and liver and spleen enlargement. Serum biochemical parameters were creatinine, uric acid, lactate dehydrogenase (LDH), alkaline phosphatase, total bilirubin, and albumin. Peripheral blood (PB) features included hemoglobin level, platelet count, WBC count, and blast cell percentage. Bone marrow aspirate parameters evaluated were cellularity, peroxidase reactivity, blast cell percentage, French-American-British (FAB) subtype, cytogenetics, FLT3-internal tandem duplication (FLT3-ITD) mutations, and PML-RARA isoforms. The following surface antigen markers were evaluated by multiparametric flow inmunocytometry: CD2, CD7, CD11b, CD13, CD14, CD15, CD19, CD34, CD56, and CD117. Positivity for a specific antigen was defined as expression of the corresponding surface marker by more than $20 \%$ of blast cells. Other variables such as PETHEMA LPA trial (LPA96 vs LPA99), fibrinogen level, and the presence of coagulopathy were also included. Coagulopathy was defined as a prolonged prothrombin time and/or activated partial thromboplastin time in addition to hypofibrinogenemia and/or increased levels of fibrin degradation products or D-dimer. The prognostic impact of DS on induction mortality and risk of relapse was also analyzed.

\section{Statistical methods}

Chi-square with Yates' correction, when appropriate, was used to analyze differences in the distribution of variables among patient subsets. The Student $t$ test and Mann-Whitney $\mathrm{U}$ test were used to compare continuous variables with normal and nonnormal distribution, respectively. $P$ values were calculated using the 2-tailed test. Characteristics selected for inclusion in the multivariate analysis were those for which there was some indication of a significant association with DS in univariate analysis $(P<.1)$ and, if available, those for which prior studies had suggested a possible relationship. Multivariate analysis was performed using a logistic regression model. ${ }^{18}$ Missing data were substituted by the mean values from patients for whom data were available. ${ }^{19}$ For univariate comparison, unadjusted time-toevent analyses (relapse-free survival [RFS]) were performed using the Kaplan-Meier estimate, ${ }^{20}$ as well as log-rank tests and their generalizations. ${ }^{21}$ Patient follow-up was updated in February 2008 and the median follow-up of the cohort was 80 months (range, 29-133 months). Computations were performed using $4 \mathrm{~F}, 3 \mathrm{D}, \mathrm{LR}, 1 \mathrm{~L}$, and $2 \mathrm{~L}$ programs from the BMDP statistical library (BMDP Statistical Software, Los Angeles, CA).22

\section{Results}

\section{Accrual and patient characteristics}

Between November 1996 and June 2005, 793 consecutive patients with a genetic diagnosis of APL were registered from 82 institutions in Spain, The Netherlands, Belgium, Argentina, Uruguay, and 
Table 1. Initial characteristics of patients with and without differentiation syndrome (DS)

\begin{tabular}{|c|c|c|c|c|c|c|c|}
\hline \multirow[b]{2}{*}{ Characteristic } & \multicolumn{2}{|c|}{ DS } & \multicolumn{2}{|c|}{ No DS } & \multirow[b]{2}{*}{$P$} & \multicolumn{2}{|c|}{ Total } \\
\hline & $\begin{array}{l}\text { Median } \\
\text { (range) }\end{array}$ & No. (\%) & $\begin{array}{l}\text { Median } \\
\text { (range) }\end{array}$ & No. (\%) & & $\begin{array}{l}\text { Median } \\
\text { (range) }\end{array}$ & No. (\%) \\
\hline Overall & & $183(100)$ & & $556(100)$ & & & $739(100)$ \\
\hline \multicolumn{8}{|l|}{ Protocol } \\
\hline LPA96 & & $53(29)$ & & $122(22)$ & .05 & & $175(24)$ \\
\hline LPA99 & & $130(71)$ & & $434(78)$ & & & $564(76)$ \\
\hline Age, y & $43(3-78)$ & & $39(2-83)$ & & .04 & $40(2-83)$ & \\
\hline Younger than 19 & & $17(9)$ & & $67(12)$ & & & $84(11)$ \\
\hline $19-50$ & & $103(56)$ & & $326(58)$ & & & $429(58)$ \\
\hline $51-60$ & & $24(13)$ & & $71(13)$ & & & $95(13)$ \\
\hline $61-70$ & & $24(13)$ & & $63(11)$ & & & $87(12)$ \\
\hline Older than 70 & & $15(8)$ & & $29(5)$ & & & $44(6)$ \\
\hline \multicolumn{8}{|l|}{ Sex } \\
\hline Male & & $100(55)$ & & $274(49)$ & .21 & & $374(51)$ \\
\hline Female & & $83(45)$ & & $282(51)$ & & & $365(49)$ \\
\hline ECOG performance status & $1(0-3)$ & & $1(0-3)$ & & .32 & $1(0-3)$ & \\
\hline $0-1$ & & $123(72)$ & & $389(76)$ & & & $512(75)$ \\
\hline 2 & & $37(22)$ & & $86(17)$ & & & $123(18)$ \\
\hline 3 & & $10(6)$ & & $36(7)$ & & & $46(7)$ \\
\hline \multicolumn{8}{|l|}{ Fever } \\
\hline No & & $109(60)$ & & $338(61)$ & .71 & & $447(61)$ \\
\hline Yes & & $73(40)$ & & $212(38)$ & & & $285(39)$ \\
\hline WBC count, $\times 10^{9} / \mathrm{L}$ & $3.3(0.4-133)$ & & $2.0(0.2-460)$ & & $<.001$ & $2.2(0.2-460)$ & \\
\hline Less than 2 & & $69(38)$ & & $281(51)$ & & & $350(47)$ \\
\hline $2-5$ & & $35(19)$ & & $105(19)$ & & & $140(19)$ \\
\hline $5-10$ & & $16(9)$ & & $46(8)$ & & & $62(8)$ \\
\hline $10-50$ & & $48(26)$ & & $85(15)$ & & & $133(18)$ \\
\hline More than 50 & & $15(8)$ & & $38(7)$ & & & $53(7)$ \\
\hline Platelet count, $\times 10^{9} / \mathrm{L}$ & $20(1-207)$ & & $23(1-207)$ & & .19 & $22(1-207)$ & \\
\hline Less than 10 & & $32(17)$ & & $102(18)$ & & & $134(18)$ \\
\hline $10-40$ & & $116(59)$ & & $318(67)$ & & & $434(59)$ \\
\hline More than 40 & & $35(19)$ & & $135(24)$ & & & $170(23)$ \\
\hline Hemoglobin, g/dL & $9.5(4-16.9)$ & & $9.0(3-15.9)$ & & .12 & $9.3(3-16.9)$ & \\
\hline Less than or equal to 10 & & $112(61)$ & & $357(64)$ & & & $469(64)$ \\
\hline More than 10 & & $71(39)$ & & $198(36)$ & & & $269(36)$ \\
\hline Creatinine, $\mathrm{mg} / \mathrm{dL}$ & $1.0(0.2-2)$ & & $0.9(0.2-2.4)$ & & .03 & $0.9(0.2-2.4)$ & \\
\hline Less than or equal to 1.4 & & $170(96)$ & & $532(99)$ & & & $702(98)$ \\
\hline More than 1.4 & & $7(4)$ & & $7(1)$ & & & $14(2)$ \\
\hline Albumin, mg/dL & $4.0(2.3-6.7)$ & & $4.0(1.7-6.2)$ & & .99 & $4.0(1.7-6.7)$ & \\
\hline Less than or equal to 3.5 & & $34(23)$ & & $93(21)$ & & & $127(21)$ \\
\hline More than 3.5 & & $134(77)$ & & $356(79)$ & & & $470(79)$ \\
\hline \multicolumn{8}{|l|}{ Coagulopathy } \\
\hline No & & $31(17)$ & & $140(25)$ & .02 & & $171(23)$ \\
\hline Yes & & $151(83)$ & & $412(75)$ & & & $563(77)$ \\
\hline \multicolumn{8}{|l|}{ FAB subtype } \\
\hline Hypergranular & & $143(78)$ & & $455(83)$ & .11 & & $598(82)$ \\
\hline Microgranular & & $40(22)$ & & $91(17)$ & & & $131(18)$ \\
\hline \multicolumn{8}{|l|}{ PML-RARA isoform } \\
\hline BCR1/BCR2 & & $90(55)$ & & $292(59)$ & .35 & & $382(58)$ \\
\hline BCR3 & & $73(45)$ & & $200(41)$ & & & $271(42)$ \\
\hline
\end{tabular}

the Czech Republic (the names of the institutions and clinicians involved are listed in the Appendix, available on the Blood website; see the Supplemental Materials link at the top of the online article). Forty-two patients $(5 \%, 8$ [4\%] in the LPA96 and 34 [6\%] in the LPA99 trials) were considered ineligible for the treatment because their severe clinical condition contraindicated the administration of chemotherapy. Thus, 751 patients who met the previously defined entry criteria were enrolled in the LPA96 and LPA99 studies. Twelve patients (5 of 180 in the LPA96 trial and 7 of 571 in the LPA99 trial) were not evaluated because of protocol violations during induction therapy. Details of ineligible and nonevaluable patients are described elsewhere. ${ }^{12}$ LPA96 and LPA99 patients were comparable for all the baseline characteristics except for sex and serum albumin levels (female sex and low albumin levels were more frequent in the LPA99 trial; both $P=.01$ ).

\section{Incidence and characteristics of patients with DS}

Overall, 183 of 739 patients (24.8\%) experienced DS; 93 (12.6\%) were severe cases and $90(12.2 \%)$ were moderate cases. In 33 patients (4.5\%) with possible DS, an unambiguous diagnosis of DS could not be made. This was due to the presence of concurrent medical problems that could explain the clinical manifestations. These problems were pulmonary hemorrhage in 13 patients, pneumonia in 8 , congestive heart failure in 6 , renal failure in 3 , and septic shock in 3 . 
Table 2. Clinical signs and symptoms of moderate and severe DS

\begin{tabular}{|c|c|c|c|c|c|c|}
\hline \multirow[b]{2}{*}{$\begin{array}{l}\text { Clinical signs and } \\
\text { symptoms }\end{array}$} & \multicolumn{6}{|c|}{ Frequency of the different clinical signs and symptoms according to the type of DS } \\
\hline & $\begin{array}{c}\text { Moderate DS } \\
(\mathrm{n}=90), \text { no. }(\%)\end{array}$ & $\begin{array}{c}\text { Severe DS } \\
(\mathrm{n}=93), \text { no. }(\%)\end{array}$ & $P^{\star}$ & $\begin{array}{l}\text { Early severe DS } \\
(n=43), \text { no. }(\%)\end{array}$ & $\begin{array}{l}\text { Late severe DS } \\
(\mathrm{n}=43), \text { no. }(\%)\end{array}$ & $P+$ \\
\hline Dyspnea & $53(59)$ & $88(95)$ & $<.001$ & $48(96)$ & $40(93)$ & .53 \\
\hline Pulmonary infiltrates per X-ray & $34(38)$ & $75(81)$ & $<.001$ & $43(86)$ & $32(74)$ & .16 \\
\hline Edema & $48(53)$ & $75(81)$ & .001 & $42(84)$ & $33(77)$ & .38 \\
\hline Unexplained fever & $48(53)$ & $69(74)$ & .003 & $34(68)$ & $35(81)$ & .14 \\
\hline Weight gain of more than $5 \mathrm{~kg}$ & $34(38)$ & $63(68)$ & $<.001$ & $37(80)$ & $26(67)$ & .15 \\
\hline Pleural effusion & $24(27)$ & $54(58)$ & $<.001$ & $32(64)$ & $22(51)$ & .21 \\
\hline Renal failure & $8(9)$ & $43(46)$ & $<.001$ & $19(40)$ & $24(57)$ & .12 \\
\hline Hypotension & $11(12)$ & $36(39)$ & $<.001$ & $13(26)$ & $23(53)$ & .007 \\
\hline Pericardial effusion & $10(11)$ & $21(23)$ & .04 & $8(16)$ & $13(30)$ & .10 \\
\hline
\end{tabular}

${ }^{*} P$ value comparing severe DS versus moderate DS.

$\dagger P$ value comparing early severe DS versus late severe DS.

Table 1 shows that age, serum creatinine levels, and WBC counts were higher among patients who developed DS than among those who $\operatorname{did} \operatorname{not}(P=.04, P=.03$, and $P<.001$, respectively). Patients with DS presented more frequently with coagulopathy $(P=.02)$.

\section{Clinical manifestations and timing of DS}

The most frequent clinical manifestations of severe DS were dyspnea (95\%), pulmonary infiltrates $(81 \%)$, unexplained fever (74\%), weight gain of more than $5 \mathrm{~kg}(68 \%)$, pleural effusion $(58 \%)$, and renal failure $(46 \%)$. The frequencies of these symptoms were considerably lower in moderate than in severe DS (Table 2). As shown in Table 2, the frequency of hypotension in late severe DS was significantly higher than in early severe DS $(P=.007)$, whereas the frequencies of other signs and symptoms did not differ significantly between patients with late and early severe DS.

DS occurred at a median of 12 days after starting ATRA treatment (range, 0-46 days). Severe DS occurred comparatively early, at a median of 6 days, while moderate DS appeared after a median of 15 days. A bimodal time distribution of the peak incidence of DS was observed (Figure 1) in both moderate and severe DS. The first peak occurred during the first week of ATRA treatment in $47 \%$ of patients, and the second peak of new cases of DS was seen in the third week $(25 \%$ of patients). During the second week, only $5 \%$ of new cases of DS were noted. DS continued to develop in some patients in the fourth week (19\%) and after day $29(3 \%)$.

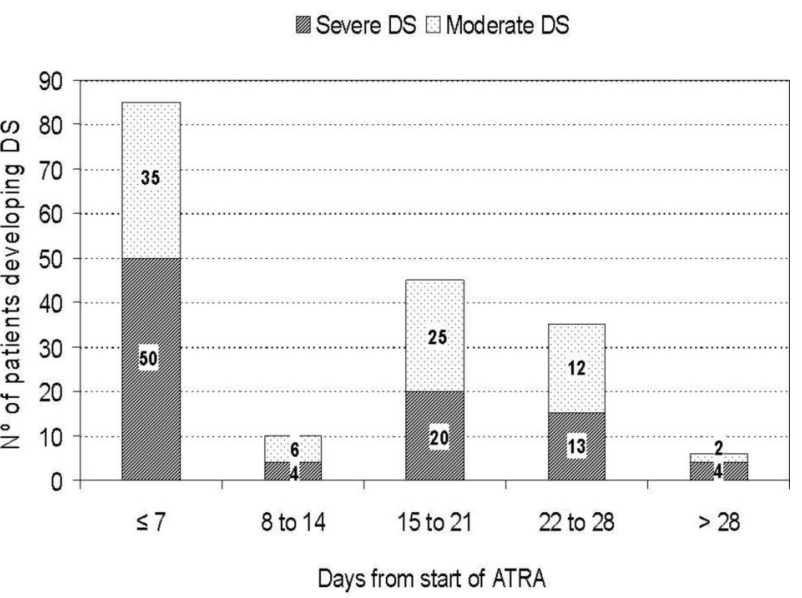

Figure 1. Time to occurrence of moderate and severe differentiation syndrome.

\section{Outcomes of DS}

A total of 667 of the 739 evaluable patients achieved morphologic CR (90.3\%; $95 \%$ confidence interval [CI], 88.0\%-92.4\%). Of the 72 induction failures, 66 patients died during induction and the remaining 6 patients were registered by their physicians as having resistant leukemia. These 6 patients were prematurely assessed as having disease refractory to induction treatment (on days 18-33 after chemotherapy), according to the classical morphologic criteria for AML. In the 6 patients, DS had been noted late (onset on days 12, 13, 15, and 15) and early (in 2 patients on day 5 ).

The development of moderate DS had no impact on the death rate during induction ( $6 \%$ with moderate DS vs $7 \%$ with no DS, $P=.82$ ), but severe DS was associated with a higher death rate during induction ( $26 \%$ with severe DS vs $7 \%$ with no DS, $P<.001$ ). The induction mortality was $40 \%$ in patients with early severe DS (Table 3). The DS-associated mortality was $11 \%$ in patients with severe DS, while no deaths resulted from moderate DS. Ten deaths related to DS occurring during induction have been described in detail elsewhere. ${ }^{12}$ Hemorrhageassociated mortality was also higher in patients who developed severe DS than in those with moderate DS or no DS (11\% vs $6 \%$ vs $4 \%$, respectively, $P=.02$ ).

Intravenous dexamethasone was administered in $90 \%$ of patients with severe DS and $82 \%$ of patients with moderate DS (Table 3). ATRA was temporarily discontinued in $64 \%$ of patients with severe DS and $60 \%$ of patients with moderate DS. Diuretics, dialysis, and mechanical ventilation were more frequently used in patients with severe DS than in those with moderate DS. Patients with severe DS required more plasma, platelet, and red blood cell transfusions during induction.

Table 3 shows that severe DS was significantly associated with higher frequencies of thrombosis and grade 3 or 4 hepatotoxicity during induction, as compared with no DS or moderate DS cases $(P=.008$ for thrombosis and $P=.01$ for grade 3 or 4 hepatotoxicity). Time to neutrophil recovery was significantly shorter in patients with severe DS $(P=.003)$. Patients with severe DS, especially those with late DS, spent more days in the hospital during induction treatment $(P=.06)$.

\section{Prognostic factors for DS}

Factors predicting moderate DS. The univariate analysis of prognostic factors identified the following characteristics as significantly associated with moderate DS: high WBC count at presentation, with $10 \times 10^{9} / \mathrm{L}$ as the most significant cutoff point; $\mathrm{LDH}$ 
Table 3. Management, complications, and outcomes of moderate and severe DS

\begin{tabular}{|c|c|c|c|c|c|c|c|}
\hline & $\begin{array}{c}\text { No DS } \\
(n=556)\end{array}$ & $\begin{array}{c}\text { Moderate } \\
\text { DS }(n=90)\end{array}$ & $\begin{array}{l}\text { Overall } \\
(n=93)\end{array}$ & $\boldsymbol{P}^{\star}$ & $\begin{array}{l}\text { Early severe } \\
\text { DS }(n=50)\end{array}$ & $\begin{array}{l}\text { Late severe } \\
\text { DS }(n=43)\end{array}$ & $P \dagger$ \\
\hline \multicolumn{8}{|l|}{ Management of DS } \\
\hline ATRA withdrawal, no. (\%) & $87(16)$ & $54(60)$ & $60(64)$ & $<.001$ & $39(78)$ & $21(49)$ & .003 \\
\hline ATRA duration shorter than 30 days, no. (\%)‡ & $100(26)$ & $36(49)$ & $36(60)$ & $<.001$ & $11(48)$ & $15(68)$ & .87 \\
\hline Intravenous dexamethasone, no. (\%) & $90(16)$ & $74(82)$ & $84(90)$ & $<.001$ & $46(92)$ & $38(88)$ & .55 \\
\hline Diuretics, no. (\%) & NA & $60(67)$ & $81(87)$ & .001 & $43(86)$ & $38(88)$ & .73 \\
\hline Dialysis, no. (\%) & NA & $1(1)$ & $11(12)$ & .003 & $8(16)$ & $3(7)$ & .18 \\
\hline Mechanical ventilation, no. (\%) & NA & $7(8)$ & $24(26)$ & .002 & $18(36)$ & $6(14)$ & .02 \\
\hline \multicolumn{8}{|l|}{ Outcome and complications of DS } \\
\hline Death during induction, no. (\%) & $37(7)$ & $5(6)$ & $24(26)$ & $<.001$ & $20(40)$ & $4(9)$ & .001 \\
\hline Death due to DS, no. (\%) & $0(0)$ & $0(0)$ & $10(11)$ & $<.001$ & $8(16)$ & $2(5)$ & .08 \\
\hline Death due to hemorrhage, no. (\%) & $22(4)$ & $5(6)$ & $10(11)$ & .02 & $9(18)$ & $1(2)$ & .04 \\
\hline Death due to infection, no. (\%) & $14(2)$ & $0(0)$ & $3(3)$ & .27 & $2(4)$ & $1(2)$ & .99 \\
\hline Thrombosis during induction, no. (\%) & $18(3)$ & $3(3)$ & $9(10)$ & .008 & $5(10)$ & $4(9)$ & .91 \\
\hline Grade 3-4 hepatotoxicity,§ no. (\%) & $24(5)$ & $5(6)$ & $11(14)$ & .01 & $5(12)$ & $6(15)$ & .65 \\
\hline Hospital days during induction, mean $\ddagger$ & 35.7 & 34.4 & 38.2 & .06 & 35.6 & 40.4 & .06 \\
\hline Days to PMNs greater than $1 \times 10^{9} / \mathrm{L}$, mean $\ddagger$ & 23.5 & 21.6 & 20.0 & .003 & 20.5 & 19.7 & .61 \\
\hline Days to platelets greater than $50 \times 10^{9} / \mathrm{L}$, mean $\ddagger$ & 18.7 & 17.8 & 19.9 & .11 & 19.3 & 20.5 & .35 \\
\hline Transfused platelet units during induction, meanł & 40.7 & 44.5 & 58.9 & .007 & 60.2 & 57.8 & .98 \\
\hline Transfused plasma units during induction, meanł & 4.4 & 10.1 & 12.7 & $<.001$ & 19.1 & 6.5 & .01 \\
\hline Transfused RBC units during induction, meanł & 9.2 & 10.3 & 12.3 & $<.001$ & 13.1 & 11.7 & .92 \\
\hline
\end{tabular}

PMNs indicates neutrophils; RBC, red blood cell; and NA, not available.

${ }^{*} P$ value comparing severe DS versus moderate DS versus no DS.

$\dagger P$ value comparing early severe DS versus late severe DS.

$\ddagger$ Excluding patients who did not achieve complete remission.

$\S$ World Health Organization gradation scale.

greater than the upper laboratory normal (ULN) values; and PB blasts greater than $70 \%$ (Table 4).

Multivariate analysis identified a WBC count greater than $10 \times 10^{9} / \mathrm{L}$ as an independent prognostic factor for moderate DS $(P=.039$; Table 5$)$.

Factors predicting severe DS. Univariate analysis identified the following prognostic factors as regards severe DS: high WBC count at presentation, with $5 \times 10^{9} / \mathrm{L}$ as the most significant cutoff point; abnormal levels of serum creatinine; FLT3-ITD mutations; microgranular FAB subtype; short PML-RARA isoform; and male sex (Table 4).

Upon multivariate analysis, abnormal levels of serum creatinine $(P=.004)$, WBC count greater than $5 \times 10^{9} / \mathrm{L}(P=.021)$, and treatment with the LPA96 protocol $(P=.044)$ remained independent prognostic factors for severe DS (Table 5).

\section{Risk of relapse of APL in patients developing DS}

The overall 7-year RFS in patients with and without DS was $85 \%$ and $87 \%$, respectively $(P=.31$; Figure $2 \mathrm{~A})$. Patients developing severe DS showed a significantly lower 7-year RFS in the LPA96 trial $(60 \%$ vs $85 \%, P=.003$; Figure $2 \mathrm{~B})$. This difference was not apparent for patients treated on the LPA99 trial (86\% vs $88 \%$; $P=.54$; Figure 2C). None of the 10 patients who had central nervous system relapse had previously experienced DS.

\section{Discussion}

This study shows that roughly one quarter of patients with APL undergoing induction therapy with the AIDA regimen develop DS, with an equal distribution of the moderate and severe forms of DS. Patients with moderate DS showed the same treatment outcome in terms of mortality during induction and relapse probability. Severe DS was associated with increased mortality during induction. A bimodal time distribution of incidence of both moderate and severe DS was observed, with peaks in the first and third week of ATRA treatment. A lower overall incidence of severe DS was observed in patients enrolled in the LPA99 trial, who systematically received prophylaxis with prednisone from the initiation of ATRA treatment for 15 days, as compared with those treated in the LPA96 trial, who received selective prophylaxis with intravenous dexamethasone when the WBC count was more than $5 \times 10^{9} / \mathrm{L}$ (before or during treatment with ATRA). A WBC count greater than $5 \times 10^{9} / \mathrm{L}$ and serum levels of creatinine greater than $1.4 \mathrm{mg} / \mathrm{dL}$ at diagnosis were identified as 2 independent factors predicting the development of severe DS. The development of severe DS correlated with a higher risk of relapse, but evidence of this relationship was restricted entirely to patients treated in the LPA96 trial, as it was not apparent in patients treated with the LPA99 protocol. It should be noted that patients with intermediate and high risks of relapse in the latter protocol received ATRA and a reinforced dose of idarubicin for consolidation, which may have contributed to the reduced relapse rate.

The variation in the incidence of DS associated with induction therapy in APL that has been previously reported is quite wide with a range from $2 \%$ to $27 \% .^{1-10}$ The impact of DS on the treatment outcomes as reported has also varied. Differences in the effects of DS between studies could be a result of several factors. The most likely explanation for different results relates to the lack of consensus in the definition, grading, and classification of the syndrome. Furthermore, the concurrent use of chemotherapy with ATRA in some but not other studies has probably determined differences in the incidence and severity of DS. In this respect, the results of a randomized study that focused on patients with low WBC counts $\left(\leq 5 \times 10^{9} / \mathrm{L}\right)^{11}$ and several nonrandomized studies $^{9,23,24}$ strongly suggest that the concurrent use of ATRA and chemotherapy decreases the incidence of DS. The GIMEMA group, ${ }^{9}$ using the AIDA regimen, reported the low incidence of DS of $2.5 \%$ ( 6 of 240 patients) but their definition of DS was notably different. They established a diagnosis of severe DS according to 
Table 4. Incidence of moderate and severe DS according to baseline characteristics of patients (univariate analysis)

\begin{tabular}{|c|c|c|c|c|c|}
\hline \multirow[b]{2}{*}{ Characteristic } & \multirow{2}{*}{$\begin{array}{c}\text { No. of } \\
\text { patients }\end{array}$} & \multicolumn{2}{|c|}{ Moderate DS } & \multicolumn{2}{|c|}{ Severe DS } \\
\hline & & No. (\%) & $P$ & No. (\%) & $P$ \\
\hline Overall & 739 & $90(12.2)$ & & $93(12.6)$ & \\
\hline \multicolumn{6}{|l|}{ Protocol } \\
\hline LPA 96 & 175 & $24(13.7)$ & .48 & $29(16.6)$ & .07 \\
\hline LPA 99 & 564 & $66(11.7)$ & & $64(11.3)$ & \\
\hline \multicolumn{6}{|l|}{ Age, y } \\
\hline Younger than 19 & 84 & $9(10.7)$ & $.18^{*}$ & $8(9.5)$ & $.29 \dagger$ \\
\hline $19-50$ & 429 & $54(12.6)$ & & $49(11.4)$ & \\
\hline $51-60$ & 95 & $6(6.3)$ & & $18(18.9)$ & \\
\hline $61-70$ & 87 & $12(13.8)$ & & $12(13.8)$ & \\
\hline Older than 70 & 44 & 9 (20.5) & & $6(13.6)$ & \\
\hline \multicolumn{6}{|l|}{ Sex } \\
\hline Male & 374 & $44(11.8)$ & .74 & $56(15.0)$ & .05 \\
\hline Female & 365 & $46(12.6)$ & & $37(10.1)$ & \\
\hline \multicolumn{6}{|l|}{ ECOG performance status } \\
\hline $0-1$ & 512 & $62(12.1)$ & .74 & $61(11.9)$ & .31 \\
\hline 2 & 123 & $16(13.0)$ & & $21(17.1)$ & \\
\hline 3 & 46 & $4(8.7)$ & & $6(13.0)$ & \\
\hline \multicolumn{6}{|l|}{ Fever } \\
\hline No & 447 & $56(12.4)$ & .81 & $53(11.8)$ & .47 \\
\hline Yes & 285 & $34(11.9)$ & & $39(13.7)$ & \\
\hline \multicolumn{6}{|l|}{ WBC count, $\times 10^{9} / \mathrm{L}$} \\
\hline Less than 2 & 350 & $37(10.6)$ & $.15 \ddagger$ & $32(9.1)$ & $.08 \S$ \\
\hline $2-5$ & 140 & $16(11.4)$ & & $19(13.6)$ & \\
\hline $5-10$ & 62 & $6(9.7)$ & & $10(16.1)$ & \\
\hline $10-50$ & 133 & $25(18.8)$ & & $23(17.4)$ & \\
\hline More than 50 & 53 & $6(11.3)$ & & $9(17.0)$ & \\
\hline \multicolumn{6}{|l|}{ Platelets, $\times 10^{9} / \mathrm{L}$} \\
\hline Less than 10 & 134 & $14(10.4)$ & .64 & $18(13.4)$ & $.36 \|$ \\
\hline $10-40$ & 434 & $57(13.1)$ & & $59(13.6)$ & \\
\hline More than 40 & 170 & $19(11.2)$ & & $16(9.4)$ & \\
\hline \multicolumn{6}{|l|}{ Coagulopathy } \\
\hline No & 171 & $14(8.2)$ & .08 & $17(9.9)$ & .24 \\
\hline Yes & 563 & $76(13.5)$ & & $75(13.3)$ & \\
\hline \multicolumn{6}{|l|}{ Creatinine, mg/dL } \\
\hline Less than or equal to 1.4 & 702 & $86(12.3)$ & .87 & $84(12.0)$ & .002 \\
\hline More than 1.4 & 14 & $1(7.1)$ & & $6(42.9)$ & \\
\hline \multicolumn{6}{|l|}{ Albumin, mg/dL } \\
\hline Less than or equal to 3.5 & 127 & $14(11.0)$ & .68 & $20(15.7)$ & .32 \\
\hline More than 3.5 & 470 & $58(12.3)$ & & $56(11.9)$ & \\
\hline \multicolumn{6}{|l|}{ LDH $\times$ ULN } \\
\hline Less than or equal to 1 & 347 & $29(8.4)$ & .004 & $38(11.0)$ & .21 \\
\hline More than 1 & 347 & $59(15.9)$ & & $49(14.1)$ & \\
\hline \multicolumn{6}{|l|}{ FAB subtype } \\
\hline Hypergranular & 598 & $74(12.4)$ & .99 & $69(11.5)$ & .05 \\
\hline Microgranular & 131 & $16(12.3)$ & & $24(18.3)$ & \\
\hline \multicolumn{6}{|l|}{ PML-RARA isoform } \\
\hline BCR1/BCR2 & 382 & $51(13.4)$ & .45 & $39(10.2)$ & .05 \\
\hline BCR3 & 273 & $31(11.4)$ & & $42(15.4)$ & \\
\hline Blasts, $\%$ of PB & & & & & \\
\hline Less than or equal to 70 & 494 & $52(10.5)$ & .02 & $61(12.3)$ & .33 \\
\hline More than 70 & 184 & $31(16.8)$ & & $28(15.2)$ & \\
\hline FLT3-ITD & & & & & \\
\hline Negative & 224 & $29(12.9)$ & .86 & $26(12.6)$ & .05 \\
\hline Positive & 66 & $8(12.1)$ & & $14(24.6)$ & \\
\hline CD2 & & & & & \\
\hline Negative & 107 & $44(13.1)$ & .29 & $43(12.8)$ & .61 \\
\hline Positive & 337 & $19(17.8)$ & & $11(10.3)$ & \\
\hline
\end{tabular}

ULN indicates upper laboratory normal (values).

${ }^{*}$ Age $>70$ years versus age $\leq 70$ years $(20.0 \%$ versus $11.7 \%, P=.09)$.

†Age $>50$ years versus age $\leq 50$ years $(15.9 \%$ versus $11.1 \%, P=.07)$

$\neq \mathrm{WBC}>10 \times 10^{9} / \mathrm{L}$ versus $\mathrm{WBC} \leq 10 \times 10^{9} / \mathrm{L}(16.7 \%$ versus $10.7 \%, P=.03)$

§WBC $>5 \times 10^{9} / \mathrm{L}$ versus WBC $\leq 5 \times 10^{9} / \mathrm{L}(16.9 \%$ versus $10.4 \%, P=.01)$.

|Platelets $>40 \times 10^{9} / \mathrm{L}$ versus platelets $\leq 40 \times 10^{9} / \mathrm{L}(13.6 \%$ versus $9.4 \%, P=.15)$. 
Table 4. Incidence of moderate and severe DS according to baseline characteristics of patients (univariate analysis) (continued

\begin{tabular}{|c|c|c|c|c|c|}
\hline \multirow[b]{2}{*}{ Characteristic } & \multirow{2}{*}{$\begin{array}{l}\text { No. of } \\
\text { patients }\end{array}$} & \multicolumn{2}{|c|}{ Moderate DS } & \multicolumn{2}{|c|}{ Severe DS } \\
\hline & & No. (\%) & $P$ & No. (\%) & $P$ \\
\hline \multicolumn{6}{|l|}{ CD11b } \\
\hline Negative & 296 & $33(11.1)$ & .69 & $34(11.5)$ & .74 \\
\hline Positive & 41 & $6(14.6)$ & & $6(14.6)$ & \\
\hline \multicolumn{6}{|l|}{ CD13 } \\
\hline Negative & 38 & $5(13.2)$ & .99 & $3(7.9)$ & .49 \\
\hline Positive & 478 & $62(13.0)$ & & $63(13.2)$ & \\
\hline \multicolumn{6}{|l|}{ CD15 } \\
\hline Negative & 322 & $46(14.3)$ & .50 & $37(11.5)$ & .59 \\
\hline Positive & 140 & $16(11.4)$ & & $13(9.3)$ & \\
\hline \multicolumn{6}{|l|}{ CD56 } \\
\hline Negative & 352 & 49 (13.9) & .99 & $39(11.1)$ & .45 \\
\hline Positive & 43 & $6(14.0)$ & & $7(16.3)$ & \\
\hline \multicolumn{6}{|l|}{ CD34 } \\
\hline Negative & 439 & $56(12.8)$ & .18 & $52(11.8)$ & .64 \\
\hline Positive & 91 & $17(18.7)$ & & $13(14.3)$ & \\
\hline
\end{tabular}

ULN indicates upper laboratory normal (values).

${ }^{*}$ Age $>70$ years versus age $\leq 70$ years $(20.0 \%$ versus $11.7 \%, P=.09)$

†Age $>50$ years versus age $\leq 50$ years $(15.9 \%$ versus $11.1 \%, P=.07)$.

¥WBC $>10 \times 10 \% / \mathrm{L}$ versus WBC $\leq 10 \times 10 \% / \mathrm{L}(16.7 \%$ versus $10.7 \%, P=.03)$.

$\S \mathrm{WBC}>5 \times 10^{9} / \mathrm{L}$ versus WBC $\leq 5 \times 10^{9} / \mathrm{L}(16.9 \%$ versus $10.4 \%, P=.01)$.

$\|$ Platelets $>40 \times 10^{9} / \mathrm{L}$ versus platelets $\leq 40 \times 10^{9} / \mathrm{L}(13.6 \%$ versus $9.4 \%, P=.15)$.

the presence of 5 of the 7 signs and symptoms as originally described by Frankel et al. ${ }^{1}$ As a matter of fact, when we apply the same definition to our series of 739 patients, we calculate a similar incidence of $2.6 \%$. In other published studies of concurrent ATRA/chemotherapy regimens, greater incidences of DS have been reported (11\% to $16 \%$ ), but the diagnostic criteria for the syndrome often were not specifically defined ${ }^{23,24}$ or the diagnostic criteria were less strict ( 3 of 7 signs and symptoms). ${ }^{10}$ In the present study, we first assessed the most discriminating cutoff value for predicting outcomes. We then classified the DS as moderate or severe according to the presence of 2 to 3 , or more than 3 of the 7 signs and symptoms, respectively. This distinction allowed for the assessment of the different prognostic significance of severe DS. The development of severe but not moderate DS appeared to be associated with an increased mortality. Interestingly, apart from a proportion of patients who died in the context of severe DS with apparently no other contributing cause of death, this complication was also associated with death resulting from hemorrhage. In line with this finding, a Japanese group ${ }^{25}$ reported that patients with DS were more prone to develop severe hemorrhage during induction. In the present study, we found that severe DS, especially early DS, was associated with coagulopathy and a higher use of plasma, platelet, and red blood cell transfusions during induction. The exacerbation of the procoagulant state of APL that can be observed with the development of $\mathrm{DS}^{26-29}$ may lead not only to an increased risk of hemorrhage but also to a higher incidence of thrombosis. This association of DS and activated procoagulation is also evident in the present study. On the other hand, as far as we know, a shorter period of neutropenia has not previously been reported, while the association of severe DS with an increased rate of grades 3 and 4 hepatotoxicity was also noted in the early study by Frankel et al. ${ }^{1}$ The bimodal peak of incidence that we observed in the first and third week of ATRA treatment in both moderate and severe DS is remarkable. It should be noted that cases of early severe DS more frequently required mechanical ventilation and were associated with higher mortality during induction than those occurring during the third week or beyond. In addition, early severe DS was characterized by a higher frequency of pulmonary infiltrates and weight gain, probably related to an initial rise in leukocytosis and fluid overload. In contrast, hypotension, unexplained fever, pericardial effusion, and renal failure were more frequent in late severe DS. We should emphasize that in this situation distinguishing between DS and other clinical complications, particularly sepsis, may be difficult. Therefore, in these cases, treatment of DS should be complemented with therapeutic measures addressing alternative diagnoses that could also explain the symptoms.

The study of prognostic factors for DS in patients treated with ATRA plus chemotherapy has scarcely been addressed in the literature. A study by the European APL Group ${ }^{10}$ analyzed prognostic factors of DS in a significant number of patients who were randomized to receive either ATRA followed by chemotherapy or both simultaneously. That study did not reveal any significant prognostic factor for DS (defined as the presence of at least 3 of 7 clinical manifestations) in any of the treatment arms. In the current study, we report several pretreatment variables predictive of the severe form of the syndrome,

Table 5. Multivariate analysis of factors predicting the occurrence of DS

\begin{tabular}{|c|c|c|c|c|c|c|c|}
\hline \multirow[b]{2}{*}{ Covariate } & \multirow{2}{*}{$\begin{array}{c}\text { Unfavorable } \\
\text { category }\end{array}$} & \multicolumn{2}{|c|}{ Overall DS } & \multicolumn{2}{|c|}{ Moderate DS } & \multicolumn{2}{|c|}{ Severe DS } \\
\hline & & OR $(95 \% \mathrm{Cl})$ & $P$ & OR $(95 \% \mathrm{Cl})$ & $P$ & OR (95\% Cl) & $P$ \\
\hline WBC count & $>5 \times 10^{9} / \mathrm{L}$ & & & & & $1.8(1.1-2.7)$ & .021 \\
\hline WBC count & $>10 \times 10^{9} / \mathrm{L}$ & $1.85(1.3-2.7)$ & .012 & $1.7(1.04-2.7)$ & .039 & & \\
\hline Creatinine & $>1.4 \mathrm{mg} / \mathrm{dL}$ & & & & & $5.8(1.9-16.9)$ & .004 \\
\hline Protocol & LPA96 & & & & & $1.7(1.04-2.7)$ & .044 \\
\hline
\end{tabular}

OR indicates odds ratio 
A

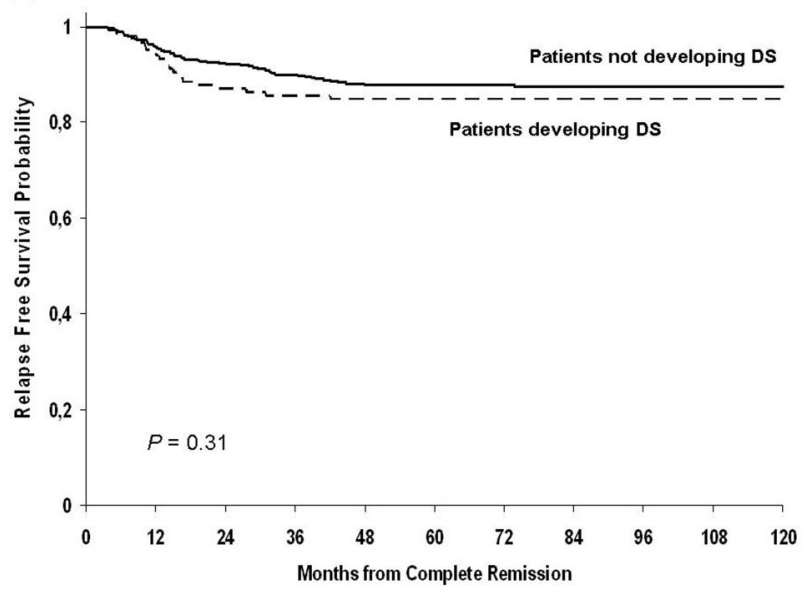

B

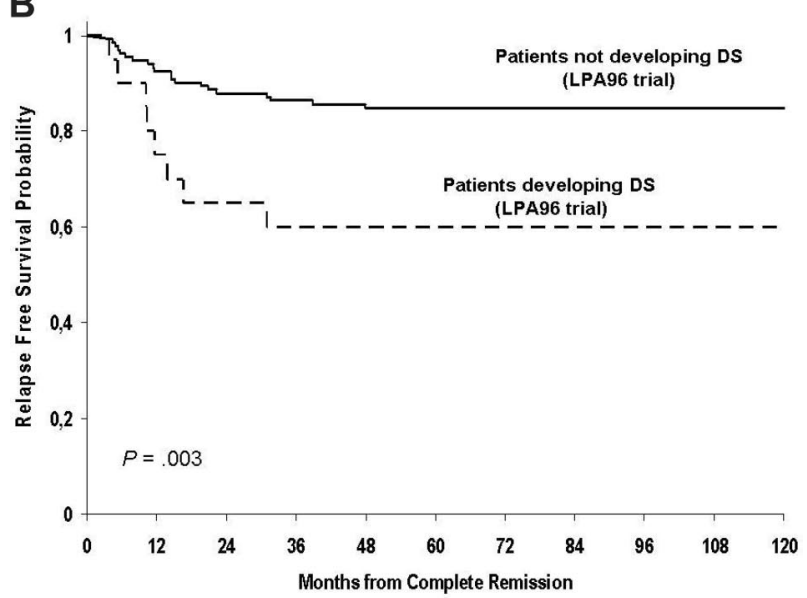

C

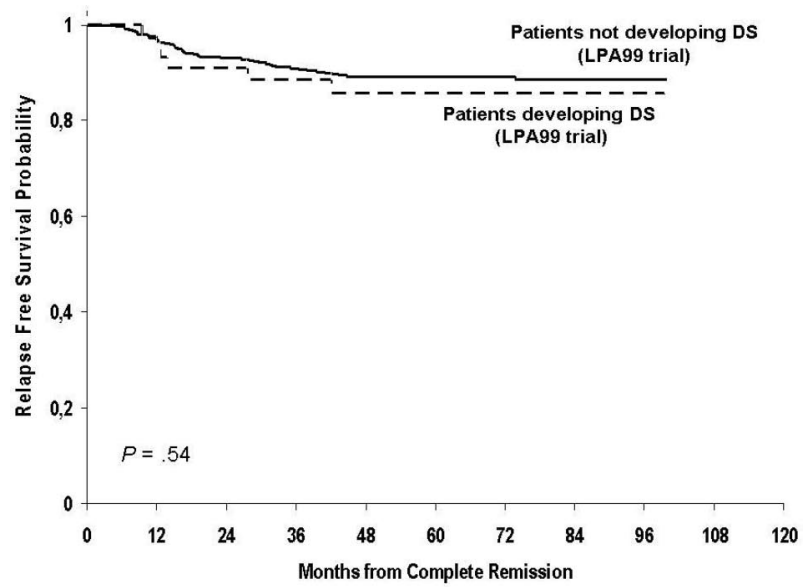

Figure 2. Kaplan-Meier estimates of relapse-free survival depending on development of differentiation syndrome during induction. (A) In both PETHEMA LPA96 and LPA99 trials; (B) in the PETHEMA LPA 96 trial; (C) in the PETHEMA LPA 99 trial.

including WBC count $\left(>5 \times 10^{9} / \mathrm{L}\right)$, abnormal serum creatinine levels, the FLT3-ITD mutation, the microgranular FAB subtype, the short PML-RARA isoform, and male sex. The significant variables were reduced to WBC counts and serum creatinine levels in multivariate analysis. None of these factors have been previously reported as predictive of DS. With regard to an abnormal levels of serum creatinine as an independent predictive factor of severe DS, this finding should be cautiously interpreted due to the small proportion of patients who had this clinical complication (6 of 90 patients with this data available who developed the severe form of the syndrome). Renal failure due, in part, to the capillary leak syndrome caused by cytokine release in DS may explain the association between high serum creatinine and severe DS. On the other hand, results of the US Intergroup study indicated that the microgranular subtype of APL protected against the development of DS. ${ }^{8}$ Patients in that study, however, had been treated with ATRA alone. With respect to presenting WBC counts, the European APL trial showed that patients with greater than $5 \times 10^{9} / \mathrm{L}$ and DS tended to need mechanical ventilation more frequently than those with lower WBC counts and DS, but this was not statistically significant and the WBC count was not associated with the development of DS. ${ }^{10}$ Apart from a mere statistical finding, we have no explanation for the paradoxical lower cutoff point of presenting WBC counts to predict severe DS $\left(5 \times 10^{9} / \mathrm{L}\right)$ compared with moderate DS $\left(10 \times 10^{9} / \mathrm{L}\right)$. Clinical and experimental studies have suggested an association between the development of DS and the expression of adhesion molecules such as $\mathrm{CD} 13^{2}$ and CD11b. ${ }^{30,31}$ We found no relationship between the expression of any of the surface markers analyzed and the development of DS.

Although the preemptive use of corticosteroids at the earliest clinical manifestations of DS has been adopted as the standard management, ${ }^{32}$ their use as prophylaxis is more controversial. The only available evidence supporting the use of corticosteroid prophylaxis is based on a study by Wiley and Firkin ${ }^{7}$ that was performed in 19 patients treated with ATRA alone. This study showed a reduction in pulmonary toxicity by administration of prednisone when the WBC count rose above $10 \times 10^{9} / \mathrm{L}$. Probably based on these results, several trials have included prophylaxis with dexamethasone in patients with more than 5 to $10 \times 10^{9} / \mathrm{L} .{ }^{23,33} \mathrm{We}$ compared the outcome following a preemptive prophylactic strategy as used in the PETHEMA LPA96 trial with the systematic prophylactic approach of the LPA99 trial, in which all patients received prednisone from the start of ATRA, regardless of WBC count. The retrospective analysis demonstrated a statistically significant reduction in the incidence of severe DS, but not in DS-related mortality, ${ }^{12}$ in relation to the systematic use of prednisone during an early 15 day interval.

The European APL Group has reported an increased relapse rate in patients developing DS. ${ }^{10,11}$ We have confirmed this finding in patients developing severe DS treated according the LPA96 trial, but not in those enrolled in the LPA99 trial. In the latter trial, however, patients with intermediate and high risks of relapse received ATRA and a reinforced dose of idarubicin for consolidation, which may have contributed to the reduced relapse rate. Finally, we found no association between DS and central nervous system relapse, as suggested by others..$^{10,34}$

In conclusion, DS is a relatively frequent and serious complication in patients with APL undergoing induction with ATRA plus chemotherapy. Prophylaxis with prednisone has no impact on reducing mortality resulting from DS, but it is likely to reduce the incidence of severe DS. Although the question of whether the use of corticosteroid prophylaxis is advantageous should be definitively settled in randomized studies, this strategy can be considered in patients treated with ATRA plus chemotherapy, particularly taking into account that infectious mortality was not apparently increased when prednisone prophylaxis was systematically used. ${ }^{12}$ Due to the association of severe DS with an increase in morbidity and mortality during induction, risk-adapted strategies focused on patients with adverse 
risk factors $\left(\mathrm{WBC}\right.$ counts $>5 \times 10^{9} / \mathrm{L}$; abnormal levels of serum creatinine) deserve further research.

\section{Acknowledgments}

The authors thank Miguel Priego for data collection and management.

This study was supported in part by the Fundación para la Investigación Hospital Universitario La Fe-Ayudas Bancaja (grant 2006/0137), Red Temática de Investigación Cooperativa en Cáncer (RD06/0020/0031) Valencia, Spain.

A complete list of the institutions and clinicians involved in this study can be found in the Appendix, available on the Blood website.

\section{Authorship}

Contribution: P.M., M.S., and J.B. conceived the study and analyzed and interpreted the data; P.M., M.S., J.B., and B.L. wrote the manuscript; P.M. performed the statistical analyses; and E.V., C.R., R.P., A.L., J.E., J.S., G.M., G.D., C.R., M.G., M.T., J.D.M., J.D.G., S.N., E.A., and S.B. included data of patients treated in their institutions, reviewed the manuscript, and contributed to the final draft.

Conflict-of-interest disclosure: The authors declare no competing financial interests.

Correspondence: Miguel A. Sanz, Head of Hematology Department, University Hospital La Fe, Avenida Campanar 21, 46009 Valencia, Spain; e-mail: msanz@uv.es.

\section{References}

1. Frankel SR, Eardley A, Lauwers G, Weiss M, Warrell R. The 'retinoic acid syndrome' in acute promyelocytic leukemia. Ann Intern Med. 1992; 117:292-296.

2. Vahdat L, Maslak P, Miller WH Jr, et al. Early mortality and the retinoic acid syndrome in acute promyelocytic leukemia: impact of leukocytosis, lowdose chemotherapy, PMN/RAR-alpha isoform, and CD13 expression in patients treated with alltrans retinoic acid. Blood. 1994;84:3843-3849.

3. Cortes JE, Kantarjian H, O'Brien S, et al. All-trans retinoic acid followed by chemotherapy for salvage of refractory or relapsed acute promyelocytic leukemia. Cancer. 1994;73:2946-2952.

4. Avvisati G, Lo Coco F, Diverio D, et al. AIDA (alltrans retinoic acid + idarubicin) in newly diagnosed acute promyelocytic leukemia: a Gruppo Italiano Malattie Ematologiche Maligne dell'Adulto (GIMEMA) pilot study. Blood. 1996;88: 1390-1398.

5. Kanamaru A, Takemoto Y, Tanimoto M, et al. All-trans retinoic acid for the treatment of newly diagnosed acute promyelocytic leukemia: Japan Adult Leukemia Study Group. Blood. 1995;85:1202-1206.

6. Fenaux P, Le Deley MC, Castaigne S, et al. Effect of all transretinoic acid in newly diagnosed acute promyelocytic leukemia: results of a multicenter randomized trial. European APL 91 Group. Blood. 1993;82:3241-3249.

7. Wiley JS, Firkin FC. Reduction of pulmonary toxicity by prednisolone prophylaxis during all-trans retinoic acid treatment of acute promyelocytic leukemia: Australian Leukaemia Study Group. Leukemia. 1995;9:774-778.

8. Tallman MS, Andersen JW, Schiffer CA, et al. Clinical description of 44 patients with acute promyelocytic leukemia who developed the retinoic acid syndrome. Blood. 2000;95:90-95.

9. Mandelli F, Diverio D, Avvisati G, et al. Molecular remission in PML/RAR alpha-positive acute promyelocytic leukemia by combined all-trans retinoic acid and idarubicin (AIDA) therapy: Gruppo Italiano-Malattie Ematologiche Maligne dell'Adulto and Associazione Italiana di Ematologia ed Oncologia Pediatrica Cooperative Groups. Blood. 1997;90:1014-1021.

10. De Botton S, Dombret H, Sanz M, et al. Incidence, clinical features, and outcome of all transretinoic acid syndromes in 413 cases of newly diagnosed acute promyelocytic leukemia. Blood 1998;92:2712-2718.

11. De Botton S, Chevret S, Coiteux V, et al. Early onset of chemotherapy can reduce the incidence of ATRA syndrome in newly diagnosed acute pro- myelocytic leukemia (APL) with low white blood cell counts: results from APL 93 trial. Leukemia. 2003;17:339-342.

12. De la Serna J, Montesinos P, Vellenga E, et al. Causes and prognostic factors of remission induction failure in patients with acute promyelocytic leukemia treated with all-trans retinoic acid and idarubicin. Blood. 2008;111:3395-3402.

13. Bennett JM, Catovsky D, Daniel MT, et al. Proposals for the classification of the acute leukemias. Br J Haematol. 1976;33:451-461.

14. Bennett JM, Catovsky D, Daniel MT, et al. A variant form of hypergranular promyelocytic leukaemia (M3). Ann Intern Med. 1980;92:280-288.

15. Sanz MA, Martín G, González M, et al. Riskadapted treatment of acute promyelocytic leukemia with all-trans retinoic acid and anthracycline monochemotherapy: a multicenter study by the PETHEMA Group. Blood. 2004;103:1237-1243.

16. Sanz MA, Lo Coco F, Martín G, et al. Definition of relapse risk and role of nonanthracycline drugs for consolidation in patients with acute promyelocytic leukemia: a joint study of the PETHEMA and GIMEMA cooperative groups. Blood. 2000;96: 1247-1253.

17. Cheson BD, Bennett JM, Kopecky KJ, et al. International Working Group for Diagnosis, Standardization of Response Criteria, Treatment Outcomes, and Reporting Standards for Therapeutic Trials in Acute Myeloid Leukemia. Revised recommendations of the International Working Group for Diagnosis, Standardization of Response Criteria, Treatment Outcomes, and Reporting Standards for Therapeutic Trials in Acute Myeloid Leukemia. J Clin Oncol. 2003;21:4642-4649.

18. Cox DR. Analysis of Binary Data. London, United Kingdom: Methuen; 1970.

19. Lee ET. Statistical Methods for Survival Data Analysis. Belmont, CA: Wadsworth; 1980.

20. Kaplan E, Meier P. Nonparametric estimation from incomplete observation. J Am Stat Assoc. 1958;53:457-481.

21. Cox DR. Regression models and life tables. J R Stat Soc B. 1972;34:187-220.

22. Dixon WJ. BMDP Statistical Software. Berkeley, CA: University of California Press; 1990.

23. Adès $L$, Chevret $S$, Raffoux $E$, et al. Is cytarabine useful in the treatment of acute promyelocytic leukemia? Results of a randomized trial from the European Acute Promyelocytic Group. J Clin Oncol. 2006;24:5703-5710.

24. Lengfelder E, Reichert A, Schoch C, et al. Double induction strategy including high dose cytarabine in combination with all-trans retinoic acid: effects in patients with newly diagnosed acute promyelocytic leukemia: German AML Cooperative Group. Leukemia. 2000;14:1362-1370.

25. Yanada M, Matsushita T, Asou N, et al. Severe hemorrhagic complications during remission induction therapy for acute promyelocytic leukemia: incidence, risk factors, and influence on outcome. Eur J Haematol. 2007;78:213-219.

26. Falanga A, Rickles FR. Pathogenesis and management of the bleeding diathesis in acute promyelocytic leukaemia. Best Pract Res Clin Haematol. 2003;16:463-482.

27. Tallman MS, Lefèbvre P, Baine RM, et al. Effects of all-trans retinoic acid or chemotherapy on the molecular regulation of systemic blood coagulation and fibrinolysis in patients with acute promyelocytic leukemia. J Thromb Haemost. 2004;2: 1341-1350.

28. Di Noto R, Schiavone EM, Ferrara F, et al. Alltrans retinoic acid promotes a differential regulation of adhesion molecules on acute myeloid blast cells. Br J Haematol. 1996;88:247-255.

29. Larson RS, Brown DC, Sklar LA. Retinoic acid induces aggregation of the acute promyelocytic leukemia cell line NB-4 by utilization of LFA-1 and ICAM-2. Blood. 1997;90:2747-2756.

30. Wu JJ, Cantor A, Moscinski LC. $\beta 2$ Integrins are characteristically absent in acute promyelocytic leukemia and rapidly upregulated in vivo upon differentiation with all-trans retinoic acid. Leuk Res. 2007;31:49-57.

31. Cunha De Santis G, Tamarozzi MB, Sousa RB, et al. Adhesion molecules and differentiation syndrome: phenotypic and functional analysis of the effect of ATRA, As2O3, phenylbutyrate, and GCSF in acute promyelocytic leukemia. Haematologica. 2007;92:1615-1622.

32. Sanz MA, Tallman MS, Lo-Coco F. Tricks of the trade for the appropriate management of newly diagnosed acute promyelocytic leukemia. Blood 2005;10:3019-3025.

33. Sanz MA, Martín G, Rayón C, et al. A modified AIDA protocol with anthracycline-based consolidation results in high antileukemic efficacy and reduced toxicity in newly diagnosed PML/RAR $\alpha$ positive acute promyelocytic leukemia. Blood. 1999;94:3015-3021.

34. Ko BS, Tang JL, Chen YC, et al. Extramedullary relapse after all-trans retinoic acid treatment in acute promyelocytic leukemia: the occurrence of retinoic acid syndrome is a risk factor. Leukemia. 1999;13:1406-1408. 